\title{
COVID-19: NEW PARADIGMS FOR DISEASE MANAGEMENT
}

Dear Reader,

The continuing threat of Corona virus Disease 2019 (COVID-19) to global health has posed critical challenges to public health, research, medical fraternity, and governments worldwide. Following the emergence of COVID-19 in the city of Wuhan in the Hubei province of China in December 2019, the number of infected individuals has been on a constant rise outside China with more than a million positive cases. With the centre of global outbreak now shifting to Europe with Italy currently having reported the second highest number of cases $(>41,000)$ and highest mortality $(3,405)$ followed by Spain, Iran $(>19,000)$, Germany $(>17,000)$ and USA $(>14,000)$, the WHO has announced it as a global pandemic. According to the official sources, more than 300 cases have been reported in India with 7 deaths so far. Citing these statistics, several countries have halted flights from COVID-19 hit regions, imposed lockdowns in several cities, suspended major public events, shutdown public places including schools, gyms, museums, clubs, etc. and are urging their citizens to self-quarantine.

Corona viruses are comparatively large viruses consisting of a single-stranded positive-sense RNA genome encapsulated within a membrane envelope that has crown-like glycoprotein spikes on its surface, leading to the nomenclature Corona virus. They have four sub-groups viz. alpha, beta, gamma, and delta of which alpha and beta forms have been commonly found to infect humans. With its first recognition in mid-1960's, seven coronaviruses have been noted for causing human infection, latest being the beta form of novel coronavirus which causes severe acute respiratory syndrome (SARS) and is termed as SARS-CoV-2. Recent information suggest that SARS-CoV-2 is the most transmissible/contagious among all. The most common signs of infection include respiratory symptoms, fever, cough, shortness of breath and breathing difficulties. In more severe cases, infection can cause pneumonia, multiple organ failure, and even death.

Considering the fact that there are no approved treatments or vaccines for this disease, the current situation demands all hands on deck to combat this COVID-19 pandemic. As an immediate research action, the WHO has emphasized on mobilizing research on rapid point of care diagnostics for use at the community level to quickly identify and treat sick people. Currently, SARS-CoV-2 RNA in nasopharyngeal swab, oropharyngeal swab and/or sputum samples is detected by a reverse-transcription polymerase chain reaction which may take up to 48 hours for confirmation in government approved laboratories. Thus, a lot of research has gone into the development of cutting-edge testing kits that can enable rapid diagnosis of COVID-19. Several countries including the US, China, South Korea, Japan and Italy have been using testing kits that take minutes to a few hours to produce results. Blood tests/finger prick tests that can detect antibodies produced in response to infection, nasal swab tests that detect DNA given off by the corona virus, face mask tests, and breath analyzers that can detect disease-specific biomarkers like DNA, RNA, proteins and fat molecules are currently under trial for rapid detection of COVID-19. In response to the COVID-19 outbreak in India, Indian Council of Medical Research (ICMR)-National Institute of Virology (NIV), Pune served as the apex laboratory to optimize the conventional and real-time PCR assays to test the samples of suspected cases. The Department of Health Research (DHR) / ICMR has commissioned 57 laboratories for COVID-19 testing in India. In addition, following the Indian Government's decision to allow accredited private labs to test for COVID-19, the Drugs Controller General of India (DCGI) has given 8 companies (international and Indian) the test licence to carry out COVID-19 diagnostic tests in the country.

In an attempt to improve the standard of care treatment approaches and evaluate the effect of adjunctive and supportive therapies to find a befitting solution to this pandemic, multicentre clinical trials are going on in multiple countries, the information on which is being constantly updated on the International Clinical Trials Registry Platform on the WHO website. The registry has a total of 508 trials on catering to different aspects of COVID-19 in the database, as of now, with the numbers increasing each day. Severe acute respiratory failure has been the most critical condition responsible for fatal outcomes of the infection and hence major clinical trials are focusing on dealing with evaluation of different therapeutics to manage this condition. Given the lengthy process of new drug development, the current strategy for immediate treatment of COVID-19 is drug repurposing. Glucocorticoid therapy using methylprednisolone, repurposing of anti-retrovirals like lopinavir, ritonavir, ribavirin, danorevir and a combination of these with/without interferon alpha-1b are being tested for their safety and efficacy to treat the pneumonia caused by SARS-CoV-2. Remdesivir, an experimental antiviral drug is being studied in hospitalized adult patients with mild/moderate as well as severe respiratory disease. Favipiravir is another drug which is being tested for its efficacy in COVID-19 caused pneumonia. Use of mesenchymal stem cells alone and in combination with ruxolitinib, recombinant cytokine genederived protein injections, recombinant interferons, antibodies, convalescent plasma and immunoregulatory therapy are also being investigated for treating pneumonia arising from COVID-19. Chloroquine, used to treat malaria and amebiasis, and hydroxychloroquine, a less toxic metabolite of chloroquine, used to treat rheumatic diseases, are also being repurposed and investigated for COVID-19 treatment. In addition, numerous traditional Chinese medicines like Lianhua-Qingwen and Jingyebaidu capsules/granules alone, and in combination with western medicines, different 
hormone doses, and interferon atomization are also proposed for trials and form a major chunk in the prospective registration list with pending trail recruitment. One of the recent findings suggests the use of healed COVID-19 patients' plasma for treating the severe cases. Similar strategies are being followed in India too with the ICMR approving the use of retroviral drugs to treat some of the coronavirus patients after scientific scrutiny. API manufacturer Lasa SuperGenerics has teamed up with the Institute of Chemical Technology, Mumbai to develop the anti-viral drug Favipiravir, which is being tested as a treatment for COVID-19. Cipla has also come forward with Council of Scientific \& Industrial Research-Indian Institute of Chemical Technology (CSIR-IICT) to manufacture three promising anti-viral compounds (Favipiravir, Remdesivir and Baloxavir) to treat COVID-19. However, India will not be a participant in the WHO's clinical trials for COVID-19 as of now owing to the very small sample size.

Another major approach to curb this pandemic has been towards the development of a vaccine to protect against COVID-19. The first phase 1 clinical trial evaluating an investigational vaccine, called mRNA-1273, developed by The National Institute of Allergy and Infectious Diseases (NIAID) and a biotechnology company Moderna, Inc., has begun at Kaiser Permanente Washington Health Research Institute (KPWHRI) in Seattle.This open-label trial involves 45 healthy adult volunteers aged 18 to 55 years and evaluates the safety and ability to induce an immune response at different doses. As per the WHO, 41 candidate vaccines against COVID-19 are being investigated, most of them being in the pre-clinical stage. The developers of these vaccine candidates include major universities like Baylor College of Medicine, University of Oxford, University of Queensland and pharmaceutical companies like ZydusCadila, GlaxoSmithKline/Clover Biopharmaceuticals Inc., Janssen and Sanofi Pasteur to name a few. Pune-based Serum Institute of India has partnered with the US-based drug research company, Codagenix to develop a liveattenuated vaccine platform against several viral diseases including COVID-19. At the same time, it is important to remember that testing vaccines and drugs without analyzing the safety risks could bring unnecessary setbacks and discouragement during this crisis and in the future.

Every outbreak provides an opportunity to gain important information about emerging and re-emerging infectious pathogens and demands for a coordinated and multi-disciplinary approach to curb it. There is a need for constant surveillance, prompt diagnosis, and better understanding of the pathogenesis of these organisms to develop effective counter measures to avoid another pandemic. The United Nations Foundation and the Swiss Philanthropy Foundation, together with WHO has instituted the COVID-19 Solidarity Response Fund that enables private individuals, corporations and institutions anywhere in the world to come together to directly contribute to global response efforts against the COVID-19 pandemic. Several other medical research foundations like Wellcome and The Bill \& Melinda Gates Foundation, National Science Foundation etc. have set up seed funding to identify, assess, develop and scale-up treatments for COVID-19. In India, the Intensification of Research in High Priority Areas (IRHPA) program of Science and Engineering Research Board (SERB) has announced a special call for research in COVID19 and related respiratory viral infections. In addition to the current focus on repurposing of antiviral drugs, the pharmaceutical fraternity should aim for long-term drug development goals to identify inhibitors of the viral entry into host cells, viral replication or infection stages associated with SARS-CoV-2 or other related corona viruses.

As members of this huge Pharmaceutical Fraternity, it is our duty and responsibility to rise to this occasion, which is not only a national but an international crisis and contribute towards it in any capacity possible. Be it intellectual input or financial support, it is a need of the hour to work for the betterment of the society as a whole.

Dr. Vandana B. Patravale

https://doi.org/10.53879/id.57.03.p0005 Institute of Chemical Technology, Mumbai,

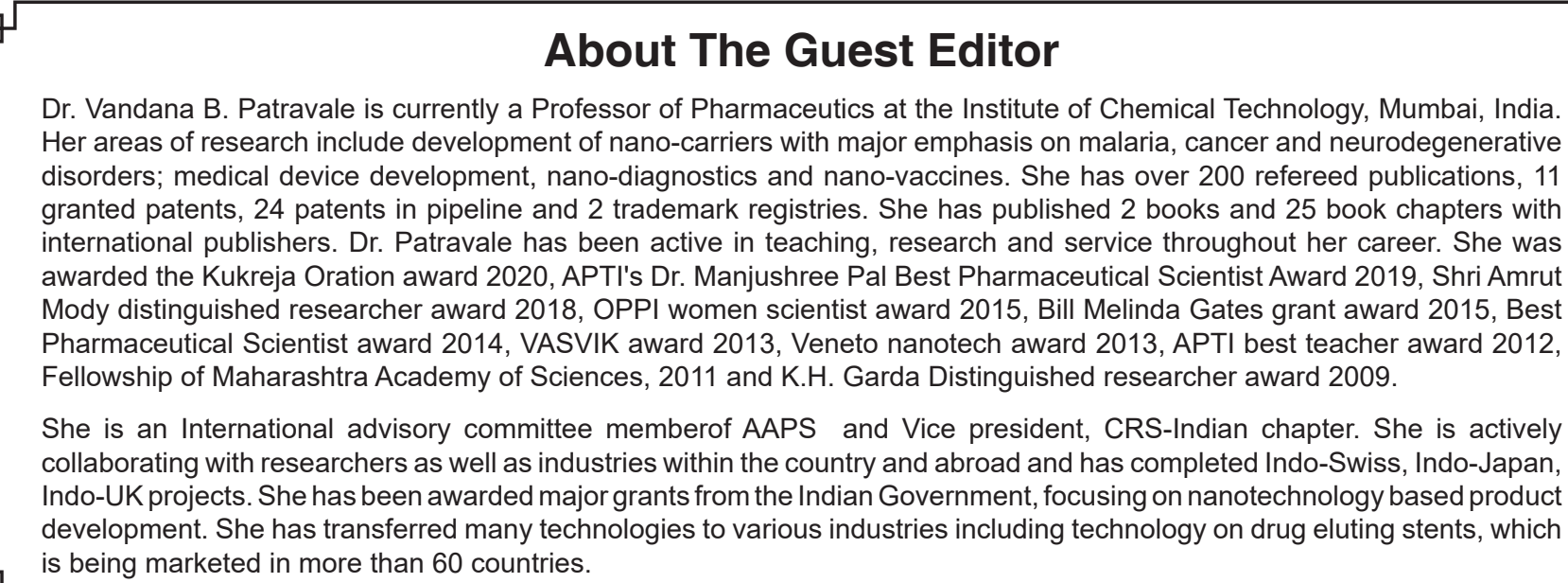

Dr. Vandana B. Patravale is currently a Professor of Pharmaceutics at the Institute of Chemical Technology, Mumbai, India. Her areas of research include development of nano-carriers with major emphasis on malaria, cancer and neurodegenerative disorders; medical device development, nano-diagnostics and nano-vaccines. She has over 200 refereed publications, 11 granted patents, 24 patents in pipeline and 2 trademark registries. She has published 2 books and 25 book chapters with international publishers. Dr. Patravale has been active in teaching, research and service throughout her career. She was awarded the Kukreja Oration award 2020, APTI's Dr. Manjushree Pal Best Pharmaceutical Scientist Award 2019, Shri Amrut Mody distinguished researcher award 2018, OPPI women scientist award 2015, Bill Melinda Gates grant award 2015, Best Pharmaceutical Scientist award 2014, VASVIK award 2013, Veneto nanotech award 2013, APTI best teacher award 2012, Fellowship of Maharashtra Academy of Sciences, 2011 and K.H. Garda Distinguished researcher award 2009.

She is an International advisory committee memberof AAPS and Vice president, CRS-Indian chapter. She is actively collaborating with researchers as well as industries within the country and abroad and has completed Indo-Swiss, Indo-Japan, Indo-UK projects. She has been awarded major grants from the Indian Government, focusing on nanotechnology based product development. She has transferred many technologies to various industries including technology on drug eluting stents, which is being marketed in more than 60 countries.

If you would like to comment on the editorial please write to us at publications@idmaindia.com 